\title{
NEW DIMENSIONS IN ANIMAL COMMUNICATION: THE CASE FOR COMPLEXITY
}

\author{
Gail L. Patricelli ${ }^{\mathrm{a}}$ and Eileen A. Hebets ${ }^{\mathrm{b}}$ \\ ${ }^{a}$ Department of Evolution and Ecology, University of California, One Shields Avenue, Davis CA \\ 95616 USA, GPatricelli@ucdavis.edu (corresponding author) \\ ${ }^{\mathrm{b}}$ School of Biological Sciences, University of Nebraska-Lincoln, 402 Manter Hall, Lincoln, NE \\ 68588 USA, ehebets2@unl.edu
}




\section{ABSTRACT}

2 Animal communication has multiple dimensions of complexity — signals may be variable over

3 time and space; signals may have multiple components interacting with each other and with the

4 receivers' perceptual and cognitive systems; and signalers and receivers may adjust their

5 behavior with changes in their environment. There is an increasing appreciation that

6 understanding animal communication — especially the diversity of signal form—requires us to

7 examine complexity per se. We review recent methods and conceptual frameworks that address

8 the complexity of communication with a specific focus on new developments in quantifying and

9 explaining signal variation, new advances in understanding receiver psychology, including

10 perceptual and cognitive biases, and new unifying frameworks, such as systems theory, which

11 provide a holistic view of complex communication systems.

\section{INTRODUCTION}

14 Many of us are drawn to study the evolution of animal communication by the mystifying

15 diversity and breathtaking beauty of animal signals, from the music of the nightingale's song, to

16 the dances of peacock spiders, to the precision of the worker bees waggling, to the vivid warning

17 colors of poison dart frogs. The traditional approach to understanding complex communication

18 involves breaking the process down into component parts - a signaler producing a signal that has

19 been selected to elicit a response from a target receiver. While an important beginning, there has

20 been a growing appreciation for the importance of addressing complexity per se in our study of

21 animal communication [1-4]. 
22 Over the last few decades, research has demonstrated that signals can be complex in multiple

23 dimensions, with interacting components in different sensory modalities, variation in form and

24 deployment over time and space, repetition and complex syntax, and conversational exchanges

25 where both parties are signalers and receivers [5-7]. Importantly, we no longer view receivers as

26 passive detectors of signals, or even optimal decision makers, as evidence accrues regarding the

27 critical role of receiver's sensory, perceptual and cognitive processes in influencing signal form

28 [8-12]. We have expanded beyond the dyad of the signaler and receiver to examine the

29 marketplace of interactors and the network of social interactions, which may shape the tactics of

30 all participants [13-16]. Extending even further, we recognize that the environmental context in

31 which communication occurs can shape the evolution of signals and responses at multiple spatial

32 and temporal scales, from brief interactions in the leaf litter to evolutionary change across

33 continents [17-21].

34 An increased appreciation of the complexity surrounding animal communication has expanded

35 the scope of our questions, encompassing more than honesty and information, allowing us to

36 begin to explain the diversity, form, and function of animal signals. Here we highlight and

37 briefly review some of the contributions from the last two years addressing new dimensions in

38 our thinking about signalers, receivers, and communication systems and we discuss areas in need

39 of further research.

\section{Dimensions of Signal Variability}

41 Whether they involve sounds, smells, colors, dynamic movements, currents, or vibrations,

42 signals often differ over time and space [18,22-25]. This variability can occur for many reasons

43 (Fig. 1), including changes in the source signal itself (e.g., changes in pitch or amplitude of 
44 songs produced) or in signaling behavior (e.g. location of singing perch), or changes due to 45 propagation through the environment (e.g. reverberation). It is increasingly clear that signal 46 variation may itself play a role in communication, for example, reflecting motor performance,

47 learning, changes in underlying condition, or tactical adjustments in signaling behavior [26-30].

48 Researchers have addressed variable signals in several ways - eliminating the variability by 49 averaging multiple measurements to represent each individual, or measuring the variability using metrics such as repeatability, consistency, coefficients of variation $(\mathrm{CV})$, or in the case of signal

51 sequences, transition probabilities. While these approaches remain useful in many cases, several

52 recent reviews discussed below have addressed limitations of these methods, proposing

53 improved methods to quantify individual variability and further explore its possible causes (Fig. $542)$.

55 Quantifying Variability- To measure and understand within-individual variation in signaling, 56 variation caused by systematic changes in conditions (e.g. experimental treatments, 57 environmental change), must be partitioned from the residual variation. Recent studies offer new 58 tools to address this challenge-Biro and Stamps [27] propose improvements in how we measure 59 repeatability, to better account for changes in behavior over time (Fig. 2), whereas Westneat et 60 al. [29] propose a statistical framework based on reaction norms to partition these types of 61 variation.

62 While studies typically focus on variation explained by experimental treatments or covariates, 63 there is growing appreciation of the interesting biology that may be hidden in the residual 64 variation [26,28,29,31]. Stamps and colleagues suggest a measure adapted from psychology that 65 describes short-term, non-systematic (or at least unexplained) variability in behavior measured 66 under the same conditions-IIV (Intra-Individual Variability) [28,31]. There is evidence in 
67 humans and other species that these 'spontaneous' endogenous fluctuations in behaviors measured as IIV may respond to selection and change with age, experience, and health [28].

69 Communicating animals may also benefit from such information, and indeed, receiver preference 70 for consistency in the performance of a signal [e.g. 32,33] may be interpreted as selection for low

71 IIV [28]. Statistically or experimentally controlling for IIV can also help to better isolate

72 predictable causes of within-individual variation in signaling behaviors, such as developmental

73 history, the physiological state of the signaler, or adaptive plasticity in response to social or 74 environmental conditions [27-29,31]. These tools and methods therefore will help to shed new 75 light on how within-individual variability in signals may reveal aspects of signaler quality as 76 well as signaling tactics.

77 Quantifying within- and among-individual variability in signals is particularly challenging when 78 signals are deployed in complex sequences. Traditional methods addressing this challenge have 79 included Markov chain analyses, permutational approaches, or entropy calculations [34], while 80 recent studies highlight new advances in sequence analysis. For example, Kershenbaum and 81 colleagues [35] call into question the common assumption that signal sequences are Markovian,

82 finding that acoustic sequences in a diverse set of taxa are better explained by non-Markovian 83 dynamics such as the 'renewal process', which tends toward repeated elements. Weiss et al. [36] 84 apply network analysis to the sequences of nightingale song types, and a drift-diffusion model 85 was used to explore how the integration of information over time influences receiver decision 86 making in grasshoppers [37]. With these recent developments, as well as increasing

87 sophistication of tools such as machine learning, and models using hidden and semi-Markovian 88 processes [34,38,39], we are compiling a robust and diverse toolkit for the analysis of sequences, 89 appropriate for the diversity of signaling systems that we study and questions that we ask. 
Adaptive Plasticity and Variation- Whether signals are repeated or produced in complex sequences, much of the dynamism in variable signals appears to reflect adaptive plasticity in signaling behavior across multiple time scales $[13,21]$. Several conceptual reviews address the causes and consequences of plasticity in signals and other traits, each with a different emphasis. among species, discussing links between plasticity and other big topics in behavioral ecology,

97 such as behavioral syndromes and animal responses to anthropogenic environmental change [41,42]. Vitousek et al. [30] emphasize dynamic feedback between signal expression, behavior, emphasizing tactical adjustments of signaling behavior to maximize benefits and decrease costs, 102 given the social and environmental context of courtship.

To understand the importance of adaptive plasticity in signaling in sexual selection, Patricelli et 104 further development, adapting models from bargaining or life history theory, or generalizing models from aggressive signaling to mate choice and other communication contexts $[13,14,26]$.

111 Ultimately, by examining the causes and consequences of within-individual variation in the expression of signals we gain a greater understanding of the scope of natural and sexual selection 
113 in shaping the diversity and complexity of signals. We are excited about the recent surge of

114 interest in this topic, providing new tools and approaches to address this understudied level of

115 variation in signaling behavior.

\section{Dimensions in Receiver Psychology}

117 While we have thus far focused our attention on signalers, inter- and intra-individual variation in

118 receivers is equally important to our understanding of animal communication. Towards this end,

119 two recent reviews aim to reignite interest in the perceptual and cognitive biases in animal

120 communication [52,53], building from the conceptual foundation established by Guilford and

121 Dawkins [8,54] and others [9,55]. Kelley and Kelley [52] use a series of fascinating examples to

122 review the importance of receiver vision and visual illusions, with a strong focus on predator-

123 prey interactions. In contrast, Ryan and Cummings' [53] review encompasses multiple sensory

124 channels, but focuses explicitly on mate choice. Both reviews emphasize the important role of

125 sensory perception and perceptual and cognitive biases in the evolution of animal

126 communication; Ryan and Cummings also warn that many of these and other drivers of signal

127 form may be missed when studies of sexual signaling focus too narrowly on indicators and signal

128 honesty. Since these reviews, a handful of empirical studies, highlighted below, have bolstered

129 their points. The receiver psychology literature has been grown rapidly, with new empirical

130 methods and with new developments in theory; a full review would be impossible in this short

131 space, but here we touch on some of the areas and ideas that we find most exciting.

132 Perceptual and Cognitive Biases- Conventional studies of receiver bias, in which researchers

133 manipulate signaling phenotypes and assess receiver responses, continue to accrue; and the

134 conceptual foundation of receiver bias, sensory drive theory [17,56], is now being applied to 
non-mate choice contexts. As one example, White and Kemp [57] highlight how sensory drive theory can help explain the colors of animal lures. Furthermore, there is increasing awareness that receiver biases can be influenced by complex interactions among dimensions of the communication system, such as sender and receiver behavior, sensory and cognitive mechanisms, and the signaling environment. For example, male great bowerbirds build bowers create forced perspective illusions in females, who stand in the bower during courtship. The field of view imposed by the bower likely increases signal efficacy through chromatic adaptation of females' eyes (which alters color perception) combined with the male's behavior of flashing colored objects into her view [58].

New research on proximate factors underlying biases in receiver sensory and cognitive systems - including genetic, biochemical, neural, and cognitive mechanisms - is combining tools and techniques from traditionally distinct fields of study to address gaps in our knowledge and challenge our assumptions. Recent studies have found that opsin gene expression in the retina, which affects color vision, differs among species, but within a species it may differ with habitat types, populations, developmental conditions, and between males and females [59-62]. Using a combination of electroretinography, morphology, and behavior, Caves et al. [63] find that cleaner shrimp are color blind and likely cannot resolve the colors and patterning of either conspecifics or client fish — critical information for understanding the evolution of morphological patterns in these animals (Fig. 3A). Yorzinski and colleagues adapted eye-tracking technology to study visual attention during mate choice in peafowl, finding that females rarely gaze at the entire upper region of the male's train. In addition, they found evidence of interactions between components, with shimmering behavior capturing and holding female's attention on the train (Fig. 3B) [64]. To date, some of the most comprehensive work on mechanisms of reproductive 
158 decision making is on poeciliid fishes. Cummings and Ramsey $[65,66]$ summarize much of this

159 work, which encompasses behavioral assays, gene expression assays, and pharmacological

160 manipulations towards the goal of understanding what they term the social cognition of mate

161 choice.

162 As researchers explore sensory capacities outside of our typical human experiences, new

163 channels of communication continue to be discovered. Thermal signaling was discovered

164 between California ground squirrels and rattle snakes [67] and air-particle displacement was

165 shown to predict agonistic interactions in amblypygids (nocturnal arachnids) [68]. Most recently,

166 polarization-mediated communication was described for northern swordtails, Xiphophorus

167 nigrensis (Fig. 3C) [69]. Examples like these, where we become aware of new communication

168 systems hiding in plain sight, dramatically illustrate the power of receiver psychology in shaping

169 animal signals.

170 Cognition and Comparative Evaluation- Bateson and Healy [9] argued that receiver decision

171 making in animal communication may be influenced by the number and type of alternatives

172 available for comparison. Such comparative evaluation has recently been shown to influence the

173 preferences of female sparrows [70], fiddler crabs [71], bushcrickets [72] and guppies, where

174 males preferentially court females surrounded by less attractive males [73]. In túngara frogs, a

175 female's binary choice between two stimulus calls is reversed in the presence of a less attractive

176 decoy call [74]. Such findings align well with behavioral economic models that incorporate

177 psychological concepts (e.g. limited cognitive resources, irrationality and sampling biases),

178 rather than traditional animal decision theory, highlighting the importance of incorporating

179 psychological concepts into our understanding of animal communication $[9,55,74-76]$. 


\section{Unifying the Dimensions of Communication}

182 The conceptual scaffoldings of the highly overlapping fields of sexual selection and animal 183 communication are rife with categorizations and dichotomies-e.g. sensory aspects of receivers 184 versus indicator models [reviewed in 20]; strategic versus tactical signal design [8]; redundant 185 versus non-redundant components [77,78]. To what extent are these categorizations and 186 dichotomies mutually exclusive? To what extent are they useful versus constraining? Exciting

187 new developments are helping to explore the common ground between theory in sexual selection 188 and signaling in other contexts [e.g. 20,57] and to integrate approaches adopted for the study of 189 complex systems into animal communication research [79].

190 Hebets and colleagues argue that the narrow redundant/non-redundant dichotomy in multimodal 191 signaling theory is not only semantically misleading, but has limited our understanding of how 192 signal complexity impacts the robustness and evolvability of behavior [79]. They use data from 193 barn swallows (Fig. 3D) [80] to highlight how complex displays may share components across 194 behavioral contexts and how within a context, overall display architecture can have structures 195 simultaneously consistent with both strategic and tactical design [79]. They bring readers from 196 the more traditional one-structure/one-function approach to an intuitive

197 multidimensional/multifunctional approach that offers a more faithful representation of complex 198 animal signaling. They argue for incorporating systems terminology, thinking, and approaches 199 into animal communication research, which integrates the concept of phenotypic integration and 200 moves from more traditional cause-and-effect thinking to synergistic thinking as it emphasizes 201 interactions and linkages [81]. 
202 A Systems Approach- In relating systems terminology and approaches into an animal

203 communication framework, Hebets and colleagues focus predominantly on the first two key

204 properties of system-level understanding [82]—quantifying the structure and dynamics of

205 signaling systems (i.e. how structure/function relationships change over time and across

206 conditions) $[79,83]$. They translate systems terminology—redundant (same structure same

207 independent function), degenerate (different structures/overlapping function), pluripotent (one

208 structure/multiple functions) — into animal communication scenarios and discuss their

209 consequences for system properties (e.g. robustness, functionality, evolvability) as well as their

210 evolutionary implications [79]. Systems-level understanding, however, also requires the

211 integration of information about the mechanistic underpinnings of system components [82]. In

212 communication systems, this goal is particularly challenging, as mechanistic underpinnings must

213 be determined and integrated both within and between two disparate systems that share varying

214 degrees of underlying machinery (e.g., genes, sensory systems) - signalers and receivers.

215 Ultimately, a system-level understanding will require the rigorous integration of data with

216 mathematical models to achieve a predictive understanding of system design principles [82,84].

217 Despite these challenges, the systems approach has the potential to help modernize animal

218 communication research — aligning it with advances in other scientific disciplines and offering

219 evolutionary hypotheses regarding the relationships between system design principles and

220 system properties. This approach provides a means for cross-taxa, cross-contextual comparisons

221 of animal signaling that can facilitate an evolutionary understanding of the multiple dimensions

222 of animal communication.

223 Visualization of complex signals - When considering multiple dimensions in communication

224 systems, visualization is a challenge. 'Phenotype networks' are a promising tool to represent the 
225 architecture of complex signals, with signal components as nodes and significant correlations

226 between components as edges (Fig. 4A). Network analysis is gaining a foothold in animal

227 communication and has been implemented in studies of birds [36,80,85] and spiders [86]. A

228 network approach allows us to go beyond pairwise metrics to quantify and compare the

229 architecture of signals across individuals and taxa, and potentially, with receiver perceptual

230 networks. Visualization and analysis of phenotype networks can reveal previously hidden

231 patterns of relationships among system components that could lead to novel hypotheses, insights,

232 and future research avenues. A similar holistic method for visualizing complex signal function

233 involves mapping behavioral outcomes into multidimensional space to generate adaptive

234 behavioral landscapes (Fig. 4B). This approach borrows from methods commonly used in

235 population genetics and evolutionary biology [overview provided in $79,87,88$ ] and is relevant to

236 testing exciting new models for divergence in sexual signals among populations [89].

237 Encompassing and integrating the full dimensionality of animal communication into our research

238 remains our most significant challenge. How do we merge a comprehensive, holistic approach to

239 animal communication (e.g. a systems approach) with within-individual variation, perceptual and

240 cognitive receiver biases, and a variable and changing signaling environment? How do we

241 simultaneously quantify, compare, and model the within- and between-system interactions of

242 signalers (e.g. genetic, biochemical and behavioral mechanisms underlying signal production)

243 and receivers (e.g., genetic, biochemical, perceptual and cognitive mechanisms underlying

244 decision making) across signaling environments and behavioral contexts (Fig. 1)? Answering

245 these questions will likely keep researchers busy for some time. 


\section{Conclusions}

248 The last few decades, and especially the last few years, have seen a great deal of progress in 249 quantifying, explaining, and modelling complexity in animal communication. We urge scientists

250 to continue to expand their conceptual frameworks beyond sexual selection theory, contest

251 theory, mimicry and aposematism, etc. to a communication framework that addresses the

252 commonalities among these contexts. We urge the development of integrated models that can

253 incorporate both sensory and indicator aspects of signal design, as well as adaptive plasticity in

254 their deployment and assessment, and which quantify and analyze communication displays

255 within the perceptual space of receivers. We see great promise in the approaches reviewed here, 256 which embrace these multiple dimensions of complexity in animal communication.

\section{ACKNOWLEDGEMENTS}

259 This work was supported by an NSF grant to GLP (IOS-1258217) and funding for EAH to 260 participate in a working group through the National Evolutionary Synthesis Center (Grant \#EF261 0905606). We thank Andy Barron, Kim Hoke, Chris Balakrishnan, Mark Hauber, Conor Taff

262 and members of the Patricelli and Hebets labs for useful discussions. For helpful discussion and 263 comments on the manuscript, we thank Alan Krakauer, Anna Perry and Ryane Logsdon. 


\section{CITATIONS}

1. Bradbury JW, Vehrencamp SL: Complexity and behavioral ecology. Behavioral Ecology 2014, 25:435-442.

2. Hebets EA, Vink CJ, Sullivan-Beckers L, Rosenthal M: The dominance of seismic signaling and selection for signal complexity in Schizocosa multimodal courtship displays. Behavioral Ecology and Sociobiology 2013, 67:1483 - 1498.

3. Ay N, Flack J, Krakauer DC: Robustness and complexity co-constructed in multimodal signalling networks. Philosophical Transactions of the Royal Society B: Biological Sciences 2007, 362:441-447.

4. Bradbury JW, Vehrencamp SL: Principles of Animal Communication, Second Edition. Sunderland, Massachusetts: Sinauer Associates; 2011.

5. Hebets EA, Papaj DR: Complex signal function: developing a framework of testable hypotheses. Behavioral Ecology and Sociobiology 2005, 57:197-214.

6. Møller AP, Pomiankowski AN: Why have birds got multiple sexual ornaments? Behavioral Ecology and Sociobiology 1993, 32:167-176.

7. Markow TA, Hanson SJ: Multivariate analysis of Drosophila courtship. Proc. Natl. Acad. Sci. U. S. A. 1981, 78:430-434.

8. Guilford T, Dawkins MS: Receiver psychology and the evolution of animal signals. Animal Behaviour 1991, 42:1-14.

9. Bateson M, Healy SD: Comparative evaluation and its implications for mate choice. Trends in Ecology \& Evolution 2005, 20:659-664.

10. Ryan MJ: Sexual Selection, Receiver Biases, and the Evolution of Sex Differences. Science 1998, 281:1999-2003.

11. Owings DH, Morton ES: Animal vocal communication: a new approach. Cambridge, United Kingdom: Cambridge University Press; 2006. 
12. Rowe C: Receiver psychology and the evolution of multicomponent signals. Animal Behaviour 1999, 58:921-931.

13. Patricelli GL, Krakauer AH, McElreath R: Assets and tactics in a mating market: economic models of negotiation offer insights into animal courtship dynamics on the lek. Current Zoology 2011, 57:225-236.

14. Noë R, Hammerstein P: Biological markets: supply and demand determine the effect of partner choice in cooperation, mutualism and mating. Behavioral Ecology and Sociobiology 1994, 35:1-11.

15. McDonald DB: Predicting fate from early connectivity in a social network. PNAS 2007, 104:10910-10914.

16. McGregor KP, Peake MT: Communication networks: social environments for receiving and signalling behaviour. acta ethologica 2000, 2:71-81.

17. Endler JA: Signals, signal conditions and the direction of evolution. The American Naturalist 1992, 139:S125-S153.

18. Rosenthal GG: Spatiotemporal Dimensions of Visual Signals in Animal Communication. Annual Review of Ecology, Evolution, and Systematics 2007, 38:155-178.

19. Marten K, Marler P: Sound transmission and its significance for animal vocalization, I. Temperate Habitats. Behavioral Ecology and Sociobiology 1977, 2:271-290.

20. Schaefer HM, Ruxton GD: Signal Diversity, Sexual Selection, and Speciation. Annual Review of Ecology, Evolution, and Systematics 2015, 46:573-592.

21. Cornwallis CK, Uller T: Towards an evolutionary ecology of sexual traits. Trends In Ecology \& Evolution 2009, 25:145-152.

22. Gerhardt HC: Female mate choice in treefrogs: static and dynamic acoustic criteria. Animal Behaviour 1991, 42:615-635.

23. Kodric-Brown A, Nicoletto PF: Female choice in the guppy (Poecilia reticulata): the interaction between color and display. Behavioral Ecology and Sociobiology 2001, 50:346-351. 
24. Bell AM, Hankison SJ, Laskowski KL: The repeatability of behaviour: a meta-analysis. Animal Behaviour 2009, 77:771-783.

25. Hutton P, Seymoure BM, McGraw KJ, Ligon RA, Simpson RK: Dynamic color communication. Current Opinion in Behavioral Sciences 2015, 6:41-49.

26. Patricelli GL, Krakauer AH, Taff CC: Variable Signals in a Complex World: Shifting Views of Within-Individual Variability in Sexual Display Traits. In Advances in the Study of Behavior. Edited by: Academic Press; 2016:319-386. vol 48.]

27. Biro PA, Stamps JA: Using repeatability to study physiological and behavioural traits: ignore time-related change at your peril. Animal Behaviour 2015, 105:223-230.

28. Stamps JA, Briffa M, Biro PA: Unpredictable animals: individual differences in intraindividual variability (IIV). Animal Behaviour 2012, 83:1325-1334.

29. Westneat DF, Wright J, Dingemanse NJ: The biology hidden inside residual within-individual phenotypic variation. Biological Reviews 2015, 90:729-743.

30. Vitousek MN, Zonana DM, Safran RJ: An integrative view of the signaling phenotype: Dynamic links between signals, physiology, behavior and social context. Current Zoology 2014, 60:739754.

31. Stamps JA: Individual differences in behavioural plasticities. Biological Reviews 2015.

32. Byers J, Hebets E, Podos J: Female mate choice based upon male motor performance. Animal Behaviour 2010, 79:771-778.

33. Byers BE: Extrapair paternity in chestnut-sided warblers is correlated with consistent vocal performance. Behavioral Ecology 2007, 18:130-136.

34. Kershenbaum A, Blumstein DT, Roch MA, Akçay Ç, Backus G, Bee MA, Bohn K, Cao Y, Carter G, Cäsar C, et al.: Acoustic sequences in non-human animals: a tutorial review and prospectus. Biological Reviews 2016, 91:13-52. 
35. Kershenbaum A, Bowles AE, Freeberg TM, Jin DZZ, Lameira AR, Bohn K: Animal vocal sequences: not the Markov chains we thought they were. Proceedings of the Royal Society BBiological Sciences 2014, 281.

36. Weiss M, Hultsch H, Adam I, Scharff C, Kipper S: The use of network analysis to study complex animal communication systems: a study on nightingale song. Proceedings of the Royal Society B-Biological Sciences 2014, 281.

37. Clemens J, Kraemer S, Ronacher B: Asymmetrical integration of sensory information during mating decisions in grasshoppers. Proceedings of the National Academy of Sciences of the United States of America 2014, 111:16562-16567.

38. Alger SJ, Larget BR, Riters LV: A novel statistical method for behaviour sequence analysis and its application to birdsong. Animal Behaviour 2016, 116:181-193.

39. Macdonald IL, Raubenheimer D: Hidden Markov Models and Animal Behaviour. Biometrical Journal 1995, 37:701-712.

40. Snell-Rood EC: An overview of the evolutionary causes and consequences of behavioural plasticity. Animal Behaviour 2013, 85:1004-1011.

41. Sih A: Frontiers on the Interface between Behavioral Syndromes and Social Behavioral Ecology. In Animal Personalities: Behavior, Physiology, and Evolution. Edited by Carere C, Maestripieri D: University of Chicago Press; 2013.

42. Sih A, Ferrari MCO, Harris DJ: Evolution and behavioural responses to human-induced rapid environmental change. Evolutionary Applications 2011, 4:367-387.

43. Sih A, Chang AT, Wey TW: Effects of behavioural type, social skill and the social environment on male mating success in water striders. Animal Behaviour 2014, 94:9-17.

44. Sullivan-Beckers L, Hebets EA: Tactical adjustment of signalling leads to increased mating success and survival. Animal Behaviour 2014, 93:111-117. 
45. White DJ, Gersick AS, Freed-Brown G, Snyder-Mackler N: The ontogeny of social skills: experimental increases in social complexity enhance reproductive success in adult cowbirds. Animal Behaviour 2010, 79:385-390.

46. Patricelli GL, Uy JAC, Walsh G, Borgia G: Sexual selection: male displays adjusted to female's response. Nature 2002, 415:279-280.

47. Patricelli GL, Krakauer AH: Tactical allocation of effort among multiple signals in sage grouse: an experiment with a robotic female. Behavioral Ecology 2010, 21:97-106.

48. Parker GA: Courtship Persistence and Female-Guarding as Male Time Investment Strategies. Behaviour 1974, 48:157-183.

49. Broom M, Ruxton GD: Some mistakes go unpunished: the evolution of "all or nothing" signalling. Evolution 2011, 65:2743-2749.

50. Johnstone RA: Honest Signalling, Perceptual Error and the Evolution of 'All-Or-Nothing' Displays. Proceedings of the Royal Society of London B: Biological Sciences 1994, 256:169-175.

51. Payne RJH, Pagel M: When is false modesty a false economy? An optimality model of escalating signals. Proceedings of the Royal Society B: Biological Sciences 1996, 263:1545-1550.

52. Kelley LA, Kelley JL: Animal visual illusion and confusion: the importance of a perceptual perspective. Behavioral Ecology 2014, 25:450-463.

53. Ryan MJ, Cummings ME: Perceptual Biases and Mate Choice. Annual Review of Ecology, Evolution, and Systematics 2013, 44:437-459.

54. Rowe C: Receiver psychology: a receiver's perspective. Animal Behaviour 2013, 85:517 - 523.

55. Shettleworth SJ: Animal cognition and animal behaviour. Animal Behaviour 2001, 61:277-286.

56. Endler JA, Basolo AL: Sensory ecology, receiver biases and sexual selection. Trends in Ecology \& Evolution 1998, 13:415-420.

57. White TE, Kemp DJ: Technicolour deceit: a sensory basis for the study of colour-based lures. Animal Behaviour 2015, 105:231-243. 
58. Endler JA, Gaburro J, Kelley LA: Visual effects in great bowerbird sexual displays and their implications for signal design. Proceedings of the Royal Society B-Biological Sciences 2014, 281.

59. Bloch NI: Evolution of opsin expression in birds driven by sexual selection and habitat. Proceedings of the Royal Society B-Biological Sciences 2015, 282.

60. Sandkam B, Young CM, Breden F: Beauty in the eyes of the beholders: colour vision is tuned to mate preference in the Trinidadian guppy (Poecilia reticulata). Molecular Ecology 2015, 24:596-609.

61. Sandkam BA, Young CM, Breden FMW, Bourne GR, Breden F: Color vision varies more among populations than among species of live-bearing fish from South America. Bmc Evolutionary Biology 2015, 15.

62. Ehlman SM, Sandkam BA, Breden F, Sih A: Developmental plasticity in vision and behavior may help guppies overcome increased turbidity. Journal of Comparative Physiology A 2015, 201:1125-1135.

63. Caves EM, Frank TM, Johnsen S: Spectral sensitivity, spatial resolution and temporal resolution and their implications for conspecific signalling in cleaner shrimp. Journal of Experimental Biology 2016, 219:597-608.

64. Yorzinski JL, Patricelli GL, Babcock JS, Pearson JM, Platt ML: Through their eyes: selective attention in peahens during courtship. The Journal of Experimental Biology 2013, 216:30353046.

65. Cummings ME, Ramsey ME: Mate choice as social cognition: predicting female behavioral and neural plasticity as a function of alternative male reproductive tactics. Current Opinion in Behavioral Sciences 2015, 6:125-131.

66. Cummings ME: The mate choice mind: studying mate preference, aversion and social cognition in the female poeciliid brain. Animal Behaviour 2015, 103:249-258. 
67. Rundus AS, Owings DH, Joshi SS, Chinn E, Giannini N: Ground squirrels use an infrared signal to deter rattlesnake predation. Proceedings Of The National Academy Of Sciences Of The United States Of America 2007, 104:14372-14376.

68. Santer RD, Hebets EA: Agonistic signals received by an arthropod filiform hair allude to the prevalence of near-field sound communication. Proceedings of the Royal Society B-Biological Sciences 2008, 275:363-368.

69. Calabrese GM, Brady PC, Gruev V, Cummings ME: Polarization signaling in swordtails alters female mate preference. Proceedings of the National Academy of Sciences of the United States of America 2014, 111:13397-13402.

70. Lyons SM, Beaulieu M, Sockman KW: Contrast influences female attraction to performancebased sexual signals in a songbird. Biology Letters 2014, 10.

71. Callander S, Jennions MD, Backwell PRY: The effect of claw size and wave rate on female choice in a fiddler crab. Journal of Ethology 2014, 30:151-155.

72. Party V, Brunel-Pons O, Greenfield MD: Priority of precedence: receiver psychology, female preference for leading calls and sexual selection in insect choruses. Animal Behaviour 2014, 87:175-185.

73. Gasparini C, Serena G, Pilastro A: Do unattractive friends make you look better? Contextdependent male mating preferences in the guppy. Proceedings of the Royal Society of London B: Biological Sciences 2013, 280.

74. Lea AM, Ryan MJ: Sexual Selection: Irrationality in mate choice revealed by tungara frogs. Science 2015, 349:964-966.

75. Akre KL, Johnsen S: Psychophysics and the evolution of behavior. Trends in Ecology \& Evolution 2014, 29:291-300.

76. Kalenscher T, Van Wingerden M: Why We Should Use Animals to Study Economic Decision Making - A Perspective. Frontiers in Neuroscience 2011, 5.

77. Partan S, Marler P: Behaviour: Communication Goes Multimodal. Science 1999, 283:1272-1273. 
78. Wiley RH: Signal detection and animal communication. Advances in the Study of Behavior 2006, 36:217-247.

79. Hebets EA, Barron AB, Balakrishnan CN, Hauber ME, Mason PH, Hoke KL: A systems approach to animal communication. Proceedings of the Royal Society B: Biological Sciences 2016, 283.

80. Wilkins MR, Shizuka D, Joseph MB, Hubbard JK, Safran RJ: Multimodal signalling in the North American barn swallow: a phenotype network approach. Proceedings of the Royal Society BBiological Sciences 2015, 282.

81. Schaefer HM, Ruxton GD: Signal Diversity, Sexual Selection, and Speciation. In Annual Review of Ecology, Evolution, and Systematics, Vol 46. Edited by Futuyma DJ; 2015:573-592. Annual Review of Ecology Evolution and Systematics, vol 46.]

82. Kitano H: Systems biology: a brief overview. Science 2002, 295:1662 - 1664.

83. Heffner HE, Heffner RS, Contos C, Ott T: Audiogram of the hooded Norway rat. Hearing Research 1994, 73:244-247.

84. Vitousek MN, Zonana DM, Safran RJ: An integrative view of the signaling phenotype: Dynamic links between signals, physiology, behavior and social context. Current Zoology 2014, 60:116.

85. Sasahara K, Cody ML, Cohen D, Taylor CE: Structural Design Principles of Complex Bird Songs: A Network-Based Approach. Plos One 2012, 7.

86. Rosenthal MF: Mating in a variable world: The implications of environmental variation for male and female mating behavior. In School of Biological Sciences. Edited by: University of Nebraska-Lincoln; 2015. vol PhD.]

87. Blows MW, Brooks R, Kraft PG: Exploring complex fitness surfaces: Multiple ornamentation and polymorphism in male guppies. Evolution 2003, 57:1622-1630.

88. Oh KP, Shaw KL: Multivariate sexual selection in a rapidly evolving speciation phenotype. Proceedings of the Royal Society of London B: Biological Sciences 2013, 280. 
465 89. Mendelson TC, Martin MD, Flaxman SM: Mutation-order divergence by sexual selection: diversification of sexual signals in similar environments as a first step in speciation. Ecology Letters 2014, 17:1053-1066.

468

469

470

471 


\section{ANNOTATIONS}

473 [26]**Patricelli et al.(2016): Synthesizes alternative hypotheses for causes of within-individual 474 variability in signals in the context of mate choice. These include 'noise', current condition

475 indicators, display refinement, and a series of hypotheses addressing tactical adjustments of

476 signaling behavior to maximize benefits and decrease costs, given the social and environmental 477 context of courtship.

478 [27]** Biro and Stamps (2015): Discusses the use of broad-sense repeatability measures (R) to 479 quantify within-individual variation in traits, including signals. Shows that most studies ignore 480 change over time and have low power for their measures of $\mathrm{R}$, and discusses problems associated 481 with these limitations. Presents suggestions for more robust measures of repeatability in future 482 studies, including the use of different metrics of $\mathrm{R}$ for different types of variability (i.e. narrow483 sense repeatability).

484 [29]**Westneat et al (2015): Presents a framework for statistically partitioning phenotypic 485 variance at multiple levels based on behavioral reaction norms, with an emphasis on within486 individual variance. Discusses processes that may influence within-individual variance, such as 487 the environment, highlighting the significance of 'residual' within-individual variation, which 488 may often include interesting biology.

489 [30]**Vitousek et al. (2015): Reviews evidence for dynamic feedbacks between physiological 490 state, signal expression, behavior, and the context and outcomes of social interactions. Discusses 491 the implications of these feedbacks for how signals evolve, how signaling phenotypes are 492 coordinated and how honesty is maintained. 
493 [31] **Stamps (2015): Presents a hierarchical framework for categorizing types of plasticities in 494 behavior-Exogenous plasticities (contextual, developmental) and endogenous plasticities 495 (circadian and circannual rhythms, IIV, and age). Discusses methods for measuring individual 496 differences in the degree of plasticity and evidence for such variation in natural systems, as well 497 as the need for research into relationships between the types of plasticities (i.e. whether some 498 individuals are more plastic in multiple contexts).

499 [34]**Kershenbaum, et al. 2014: Demonstrates using data from seven distantly related taxa 500 (including birds, bats, hyrax, whales, and primates) that the vocal sequences of many of these 501 systems are more consistent with non-Markovian renewal processes than with the traditionally 502 assumed Markovian models.

503 [40]**Snell-Rood (2013): Presents a categorization of behavioral plasticity into two major 504 forms: developmental and activational; argues that course-grained environmental variability may 505 favor the former and fine-grained environmental variability may favor the latter. Discusses how 506 knowing the mechanism of behavioral plasticity helps to predict how organisms will respond to 507 rapidly changing and novel environments.

508 [59] *Bloch (2015): Quantifies the expression of four cone opsin genes across 16 species of New 509 World warblers, finding that opsin expression is not only variable across species, but also varied 510 between males and females (i.e. signaler and receivers). In females, the expression levels of one 511 opsin (SWS2) is correlated with plumage dichromatism, suggesting the co-evolution of female 512 visual capacity and male plumage.

513 [61] *Sandkam BA, et al. (2015): Explores population level variation in opsin gene expression in 514 three species of guppy, Poecilia reticulata, $P$. parae and $P$. picta. Expression was similar 
515 between species in sympatric populations and differed across distinct populations of the same

516 species, suggesting an important role of the environment in influencing receiver perceptual

517 capacities.

518 [65]**Cummings and Ramsey: Summarizes much of the work investigating the mechanisms of 519 reproductive decision making in poeciliid fishes, which is one of the best and most

520 comprehensively studied model systems in this area of research. Encompasses behavioral assays, 521 gene expression assays, pharmacological manipulations, etc, towards the goal of understanding 522 what the authors term the social cognition of mate choice.

523 [69]*Calabrese et al. 2014: Develops novel methods to both measure polarization patterns in 524 male and female swordtails and to manipulate the polarization of the light environment and 525 assess female preferences. Finds that the polarized reflectance of females was different than that 526 of males. Females were found to spend more time in the areas with high polarization in 527 illumination, suggesting that mating preferences may be based, at least in part, on male 528 polarization patterns.

529 [74] *Lea and Ryan 2015: Highlights the importance of comparative evaluation in mate choice. 530 Female túngara frogs were given a binary choice between two call stimuli in the presence versus 531 absence of a third call type, a decoy. Two separate experiments altered the location of the decoy 532 - either on the ground between the two binary choice speakers or suspended above the ground 533 such that the call could be heard but was not accessible. In both cases, females demonstrated a 534 reversal of their preference in the presence of a decoy.

535 [79]**Hebets et al (2016): Argues for incorporating systems terminology, thinking, and 536 approaches into animal communication research. Translates systems terminology—redundant, 
537 degenerate, and pluripotent - into the context of animal communication and discusses their

538 consequences for system properties (e.g. robustness, functionality, evolvability) as well as their 539 evolutionary implications.

540 [80]*Wilkins et al. (2014): Uses 'phenotype networks' to represent the architecture of complex 541 signals in barn swallows. Here signal components as nodes and significant correlations between 542 components as edges. Allows quantification of complex relationships between components, 543 facilitating comparison between males and females and among species. 
545 Figure 1. A schematic of animal communication. Emphasizes factors that can affect signal

546 form on the evolutionary, ontogenetic or behavioral time scales.

547 Figure 2. Within-individual variation in signals. Hypothetical data illustrating some of the 548 ways in which individuals may vary over time in their signal production (in this case, signal 549 intensity). In all cases, successful signalers (high-fitness; example in blue) produce signals at a 550 higher intensity than unsuccessful signalers (low-fitness; example in orange); the sample mean is 551 a dashed line. (A) In this scenario, there is high within-individual variability but no systematic 552 change over time; the degree of variability does not differ between successful and unsuccessful 553 signalers. (B) Here, there is systematic change over time and successful signalers are more 554 consistent in their signal production than unsuccessful signalers (i.e. they have lower IIV [intra555 individual variability]). (C) Here, there is a systematic decrease over time in signal production 556 (for example, due to habituation, seasonal changes in condition, or change in the optimum signal 557 values); successful and unsuccessful signalers differ in consistency as well as slope. Traditional 558 measures of broad-sense repeatability (or consistency) would yield similar results for the high-

559 success signaler in all scenarios, yet this signaler shows higher consistency in scenarios B and C, 560 once the effect of time is removed. Biro and Stamps [26] discuss different measures of 561 repeatability for each scenario. Westneat et al. [28] discuss methods to address heterogeneous 562 within-individual residual variance, as seen in panel C. Patricelli et al. [25] discuss alternative 563 hypotheses about why sexually-selected signals may vary over time and space, with an emphasis 564 on tactical adjustments in signaling behavior.

565 Figure 3. Study systems for some recent discoveries in animal communication. (A) Caves et 566 al. 2016 [62] studied visual systems of several species of cleaner shrimp, including this species 
567 (Lysmata amboinensis) shown cleaning a moray eel, finding that they likely cannot resolve the

568 colors and patterning of either conspecifics or client fish (photo copyright CC BY-NC 2.0 by

569 Sudachi, Flickr). (B) Yorzinski et al. 2013 [63] used an eye-tracker on peafowl (Pavo cristatus) to

570 show that females focus their attention on the lower regions of the male train during mate

571 assessment (photo by Jebulon, copyright CC BY-SA 3.0). (C) Calabrese et al. [68] found

572 evidence of communication using polarized light in swordtails (Xiphophorus nigrensis)(photo

573 copyright Johnny Jensen, JJPhoto.dk). (D) Wilkins et al. 2015 [78] use phenotype networks to

574 represent the architecture of signals in barn swallows (Hirundo rustica)(photo copyright Mdf,

575 wikipedia GNU Free Documentation License 1.2).

576 Figure 4. Methods to visualize complex signals. (A) Heuristic phenotype networks illustrating

577 courtship signal architecture through component correlations for two signaling environments

578 (left and right). Data based loosely on preliminary data from the wolf spider Schizocosa

579 floridana $($ Rosenthal 2015). Node shape $=$ component type $($ circle $=$ acoustic, square $=$

580 morphological, triangle $=$ color $)$. Blue nodes represent significant predictors of copulation

581 success. Edges between nodes represent pair-wise correlations; edge thickness = correlation

582 strength. Quantitative comparisons of network architecture using density coefficients show that

583 system-wide patterns of signal degeneracy (different structure/overlapping function) change

584 across contexts. Network density (our proxy of degeneracy) is higher in the environment on the

585 left (C-values), as visualized by more and thicker connections between components. We might

586 predict then, that effective signaling in that environment is more robust to changing conditions.

587 Differences in function for components 1 and 2 across conditions may provide an example of

588 pluripotentiality - the same components serving multiple functions across signaling

589 environments. (B) Complex signals can also be visualized as response surfaces, similar to 
590 adaptive landscapes. This surface shows a hypothetical multipeaked response surface for a 591 female based on variation in two male traits. The relative height (strength) of the response peaks 592 at different trait combinations indicate female preference. Mendelson et al. 2014 [87] discuss 593 how complex, multipeaked preference surfaces, such as this example, can favor early divergence 594 among populations in sexual signals (reprinted with permission from Mendelson et al. 2014 595 [87]).

596 


\section{Signaling Environment}

\section{Abiotic Factors \\ e.g. climate, latitude and longitude, current position of sun, temperature, wind, etc.}

\section{Signaler Biology}

\section{Signal Form}

- Genetic \& biochemical architecture

- Relationship with underlying quality, e.g. parasite load, nutrition, developmental history

\section{Signal Production \&} Deployment

- Learning \& memory

- Behavioral plasticity

- Behavioral context, e.g., agonistic, alarm, reproductive

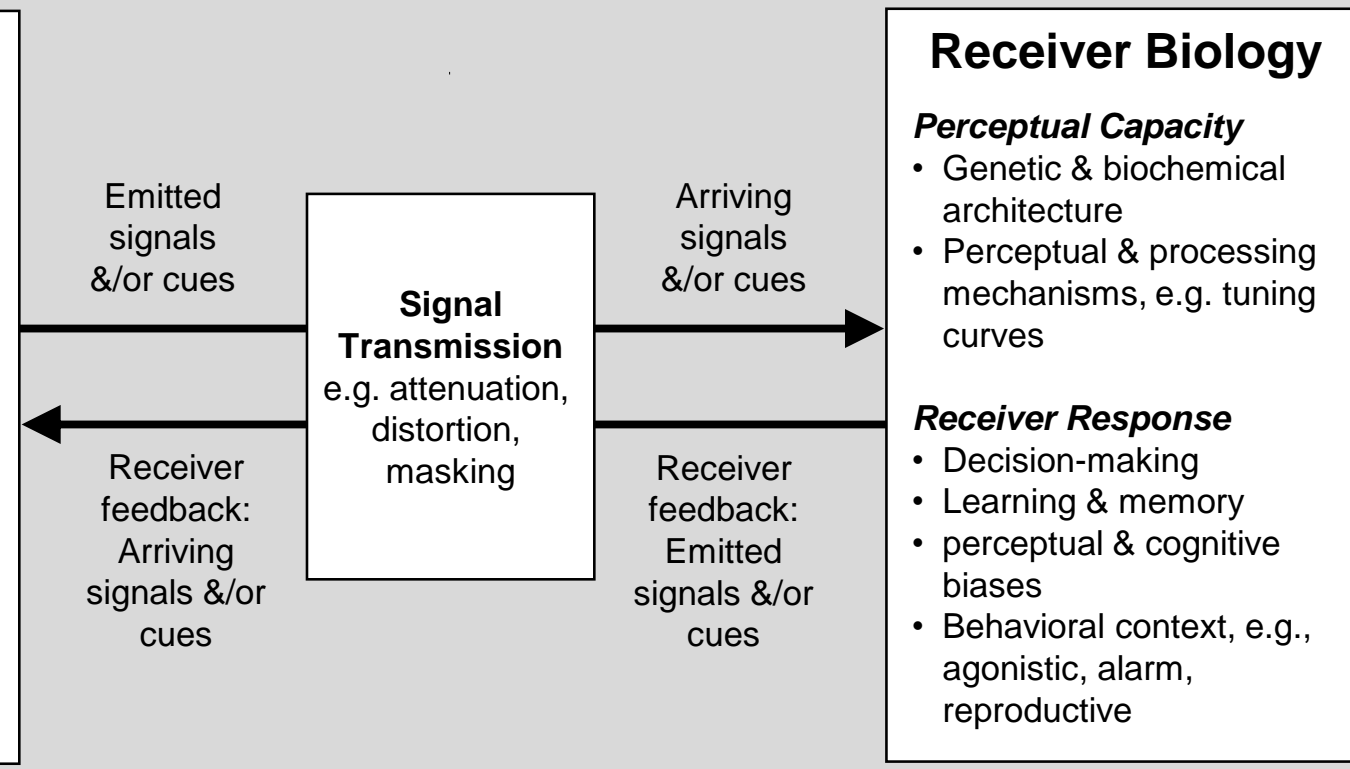

\section{Biotic Factors}

e.g. biome, food availability, predator density, surrounding vegetation, social environment such as conspecific and heterospecific signalers, etc. 


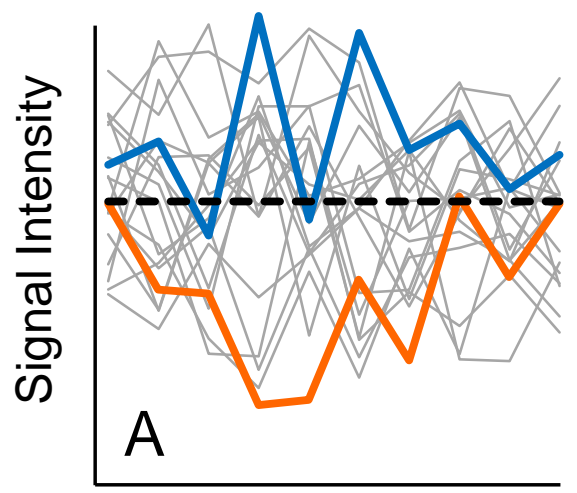

Time

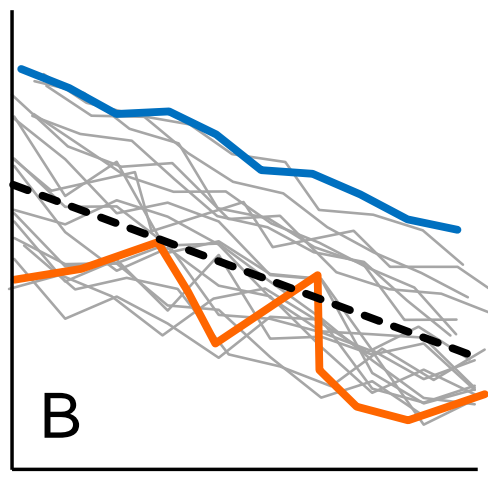

Time

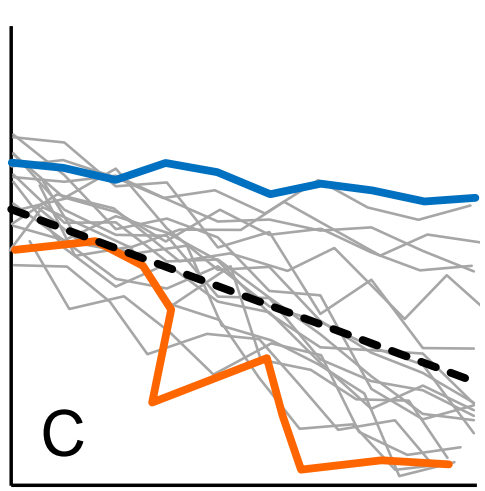

Time 

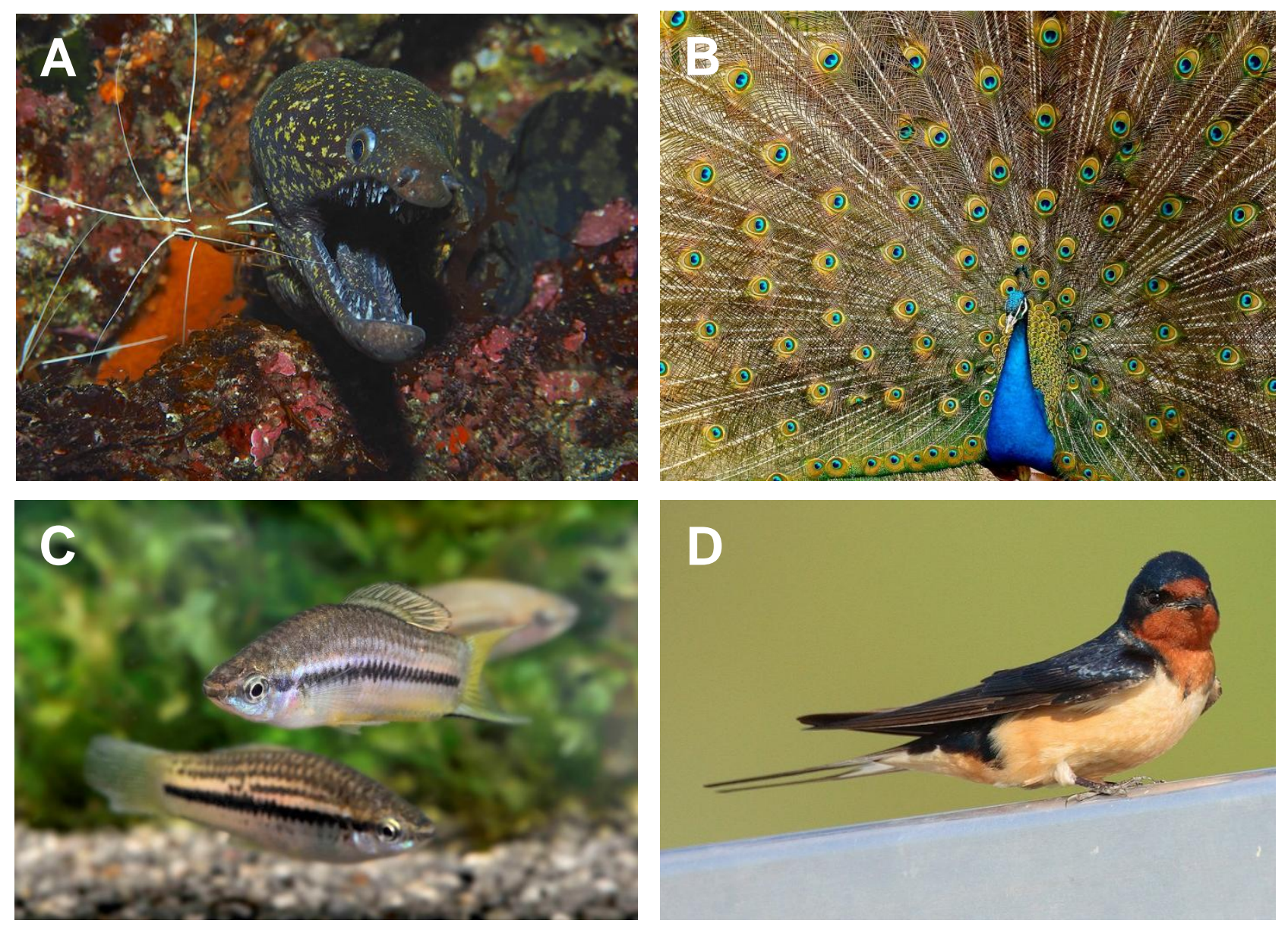

Patricelli and Hebets, Figure 3 
A
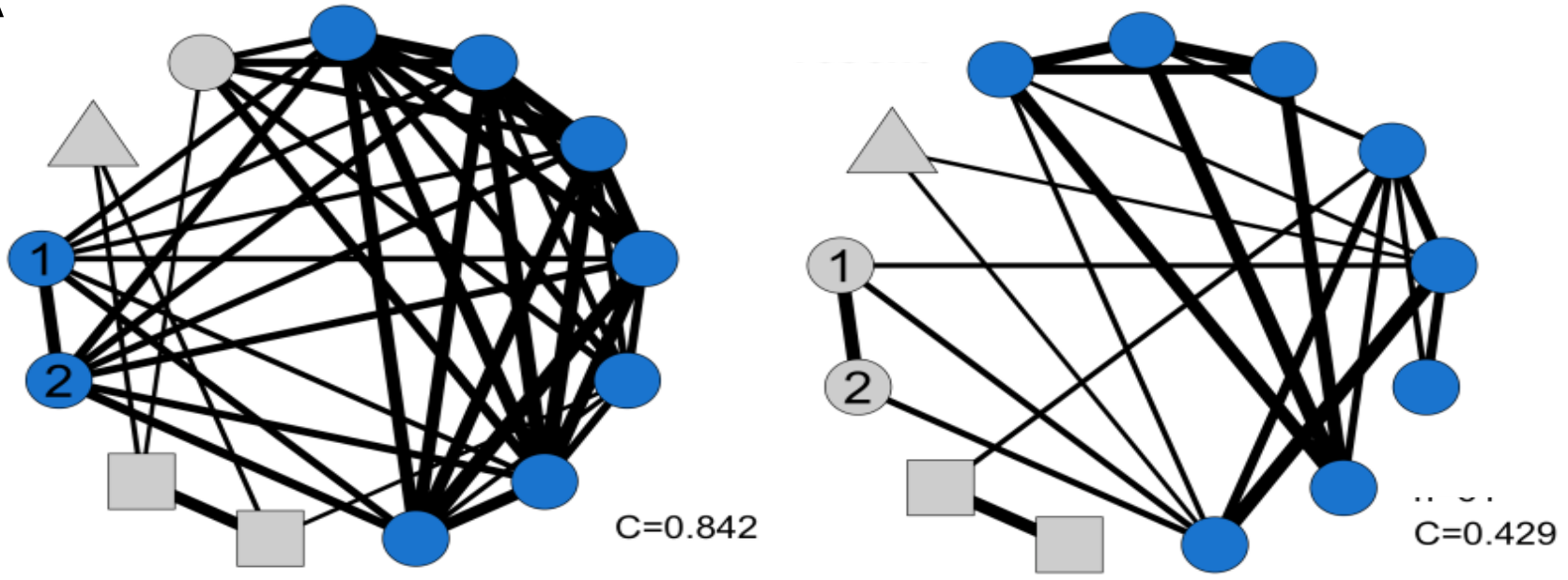

B

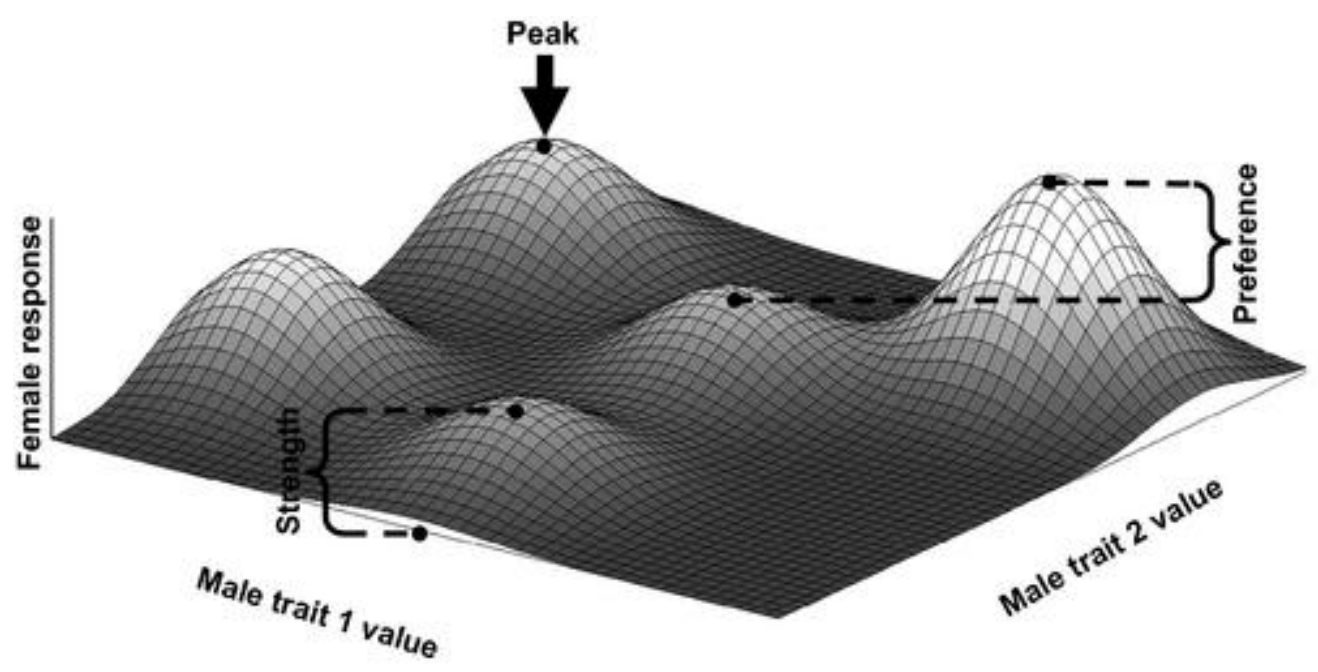

